\title{
A New Unusual Natural Pigment from Selaginella sinensis and Its Noticeable Physicochemical Properties
}

\author{
Li-Ping Zhang, Yong-Min Liang, Xiu-Cheng Wei and Dong-Liang Cheng*
}

State Key Laboratory of Applied Organic Chemistry (SKLAOC), Lanzhou University, Lanzhou 730000, P. R. China

*E-mail: chengd1@1zu.edu.cn

\section{Contents}

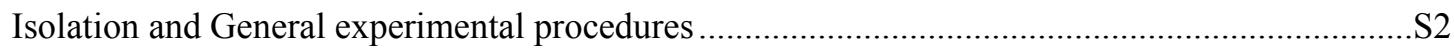

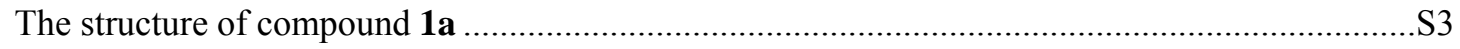

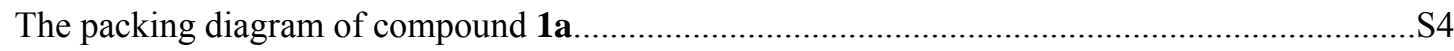

Table S1. Crystal data and structure refinement for y2038 .......................................................... 5

Table S2. Atomic coordinates and equivalent isotropic displacement parameters for y2038........S7

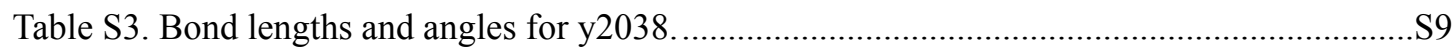

Table S4. Anisotropic displacement parameters for y2038 ........................................................ 14

Table S5. Hydrogen coordinates and isotropic displacement parameters for y2038 ......................S16

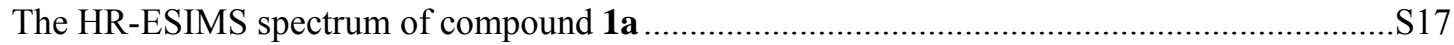

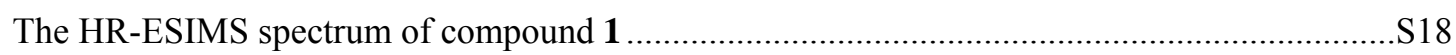

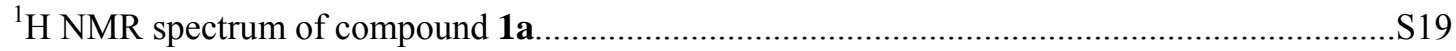

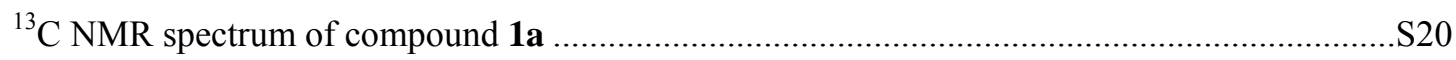

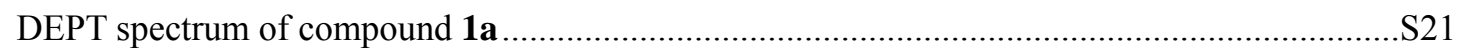

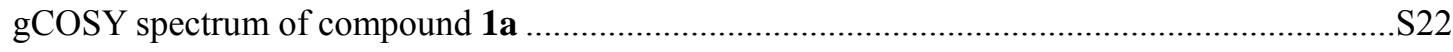

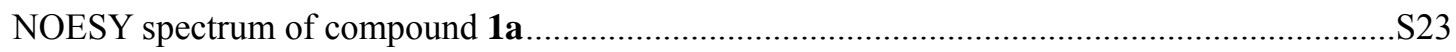

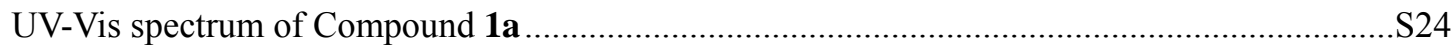

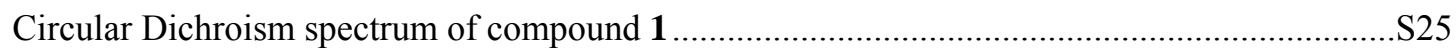

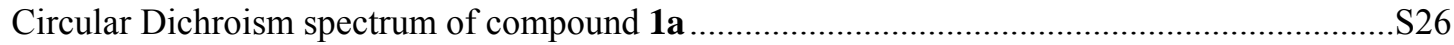




\section{Isolation}

The air-dried whole plant of Selaginella sinensis $(5.5 \mathrm{~kg})$, collected in Guangxi Autonomous Region of China in August 1999, was powdered and extracted with acetone three times at room temperature, each extraction lasting five days. The acetone solution was filtered and evaporated under reduced pressure to give an acetone extract (28 g) which was subjected to column chromatography on silica gel eluting with a gradient of petroleum/acetone (from 50:1 to 1:1) to give eight fractions (monitored by TLC analysis) from which the seventh fraction, petroleum/acetone (2:1), yielded pure Selaginellin (1) (108 mg).

General experimental procedures: Infrared spectra were recorded on a AVATAR 360 FT-IR spectrophotometer. The UV spectra were recorded on a UV-260 spectrophotometer, and the circular dichroism on DSM-1000. ${ }^{1} \mathrm{H},{ }^{13} \mathrm{C}$ NMR (DEPT) spectral data and H-H COSY, NOESY, HMQC, HMBC experiments were measured on spectrometers (300 and 400-MHz). The fluorescence spectra were recorded on a LS55 Luminescence spectrometer. 


\section{The structure of compound 1a}

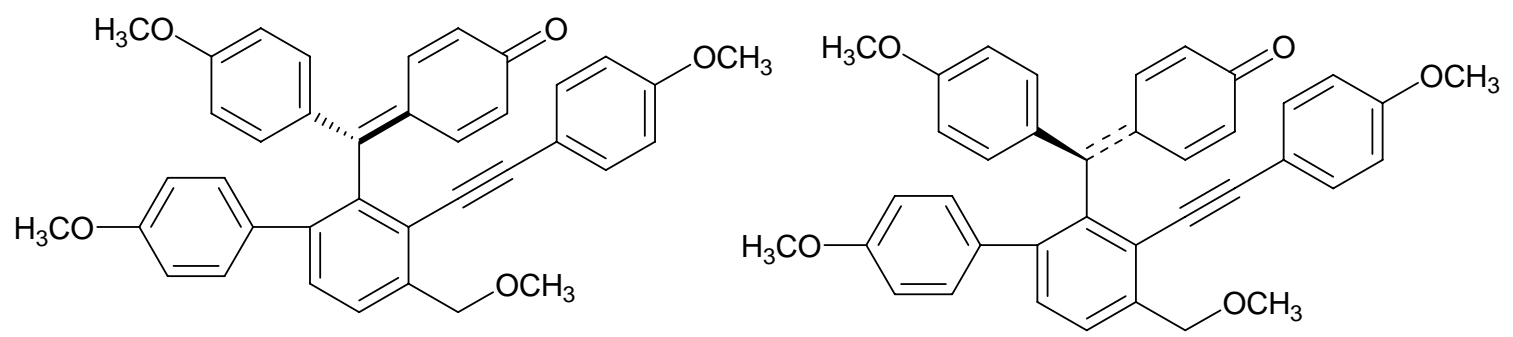

(R)-1a

(S)-1a

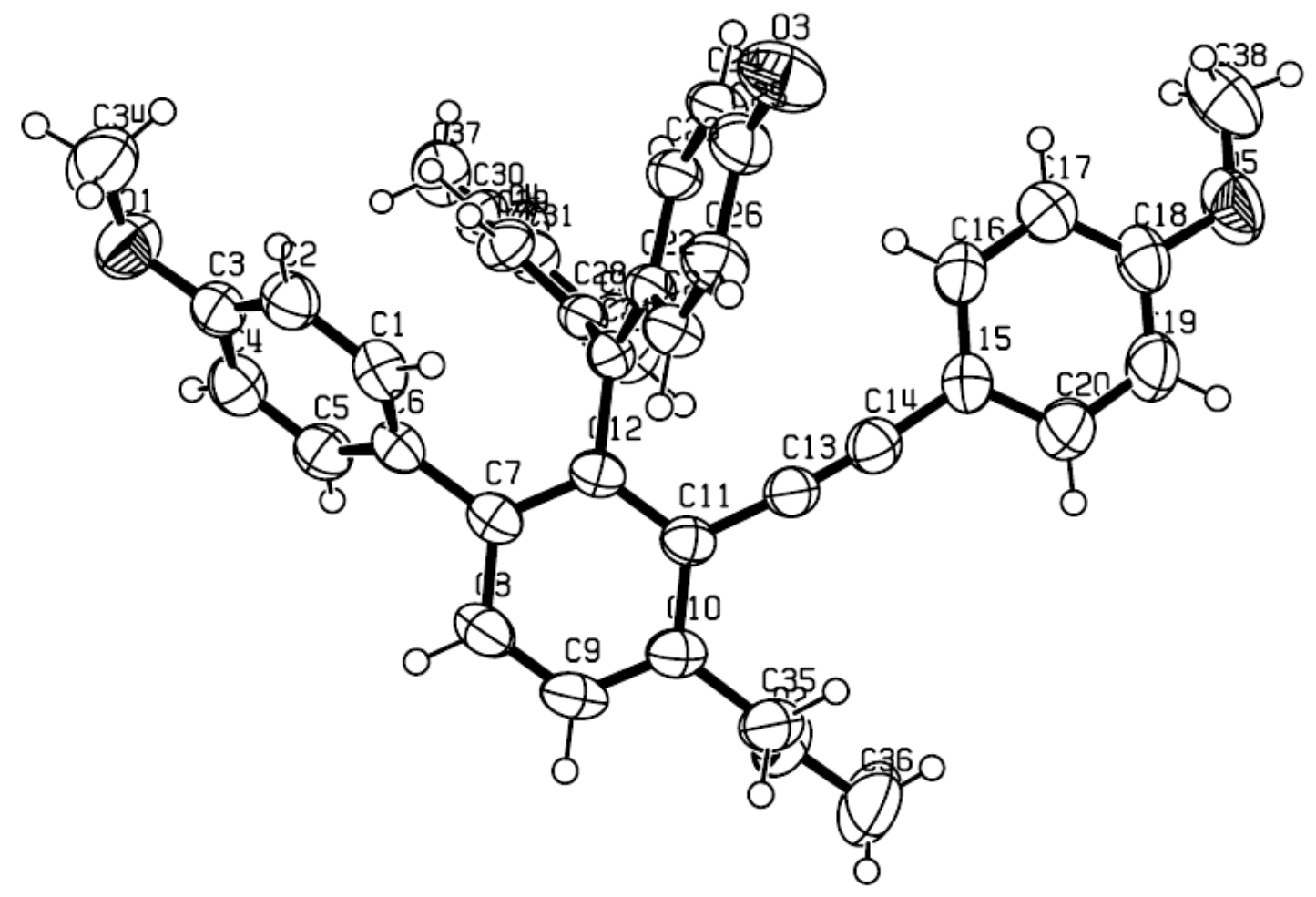

$(R)-1 \mathbf{a}$ 
The packing diagram of compound 1a

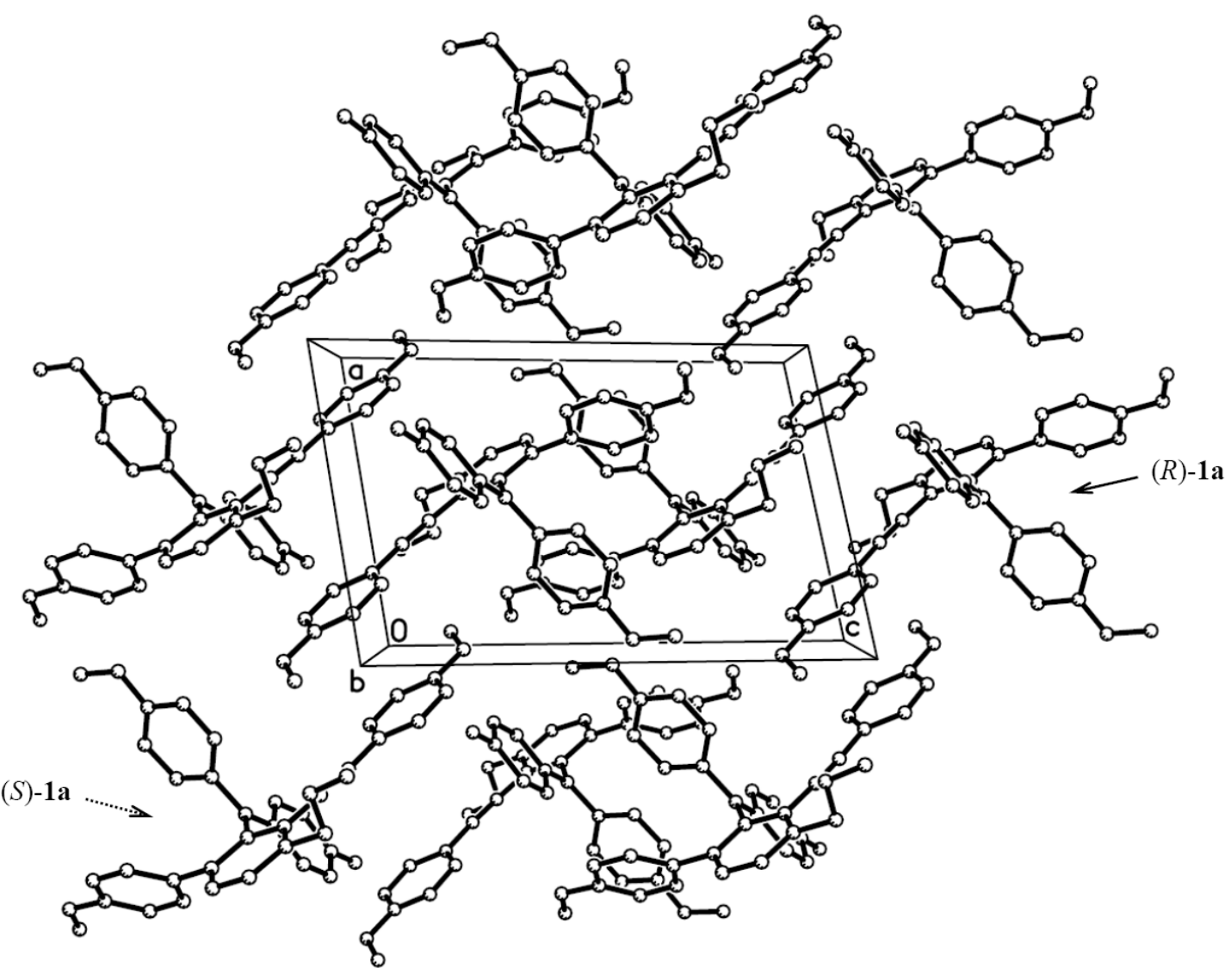

The packing diagram of compound 1a, $(R)-\mathbf{1 a}$ and $(S)$-1a are indicated by solid and dash arrows, respectively. 
Table S1. Crystal data and structure refinement for y2038.

Identification code

Empirical formula

Formula weight

Temperature

Wavelength

Crystal system

Space group

Unit cell dimensions

Volume, Z

Density (calculated)

Absorption coefficient

$\mathrm{F}(000)$

Crystal size

Theta range for data collection

Limiting indices

Reflections collected

Independent reflections

Absorption correction

Refinement method

Data / restraints / parameters y2038

C38 H32 O5

568.64

296(2) K

$0.71073 \mathrm{~A}$

Triclinic

P-1

$\mathrm{a}=9.882(2) \mathrm{A} \quad$ alpha $=103.72(1) \mathrm{deg}$.

$\mathrm{b}=11.409(2) \mathrm{A} \quad$ beta $=95.62(2) \mathrm{deg}$.

$\mathrm{c}=14.987(3) \mathrm{A} \quad$ gamma $=108.65(1) \mathrm{deg}$.

$1527.46(59) \mathrm{A}^{\wedge} 3, \quad 2$

$1.236 \mathrm{Mg} / \mathrm{m}^{\wedge} 3$

$0.081 \mathrm{~mm}^{\wedge}-1$

600

$0.56 \times 0.48 \times 0.46 \mathrm{~mm}$

1.42 to $25.50 \mathrm{deg}$.

$$
0<=\mathrm{h}<=11,-13<=\mathrm{k}<=12,-18<=\mathrm{l}<=18
$$

6180

$5629[\mathrm{R}(\mathrm{int})=0.0111]$

None

Full-matrix least-squares on $\mathrm{F}^{\wedge} 2$ 
Goodness-of-fit on $\mathrm{F}^{\wedge} 2$

Final $\mathrm{R}$ indices $[\mathrm{I}>2 \operatorname{sigma}(\mathrm{I})]$

$\mathrm{R}$ indices (all data)

Extinction coefficient

Largest diff. peak and hole
0.921

$\mathrm{R} 1=0.0369, \mathrm{wR} 2=0.0885$

$\mathrm{R} 1=0.0649, \mathrm{wR} 2=0.0951$

$0.0216(15)$

0.138 and -0.142 e. $\mathrm{A}^{\wedge}-3$ 
Table S2. Atomic coordinates $\left(x 1^{\wedge} \wedge 4\right)$ and equivalent isotropic displacement parameters $\left(A^{\wedge} 2 \times 10 \wedge 3\right)$ for $y 2038$.

$\mathrm{U}(\mathrm{eq})$ is defined as one third of the trace of the orthogonalized Uij tensor.

\begin{tabular}{|c|c|c|c|c|}
\hline & $\mathrm{x}$ & $\mathrm{y}$ & $\mathrm{z}$ & $\mathrm{U}(\mathrm{eq})$ \\
\hline $\mathrm{O}(1)$ & $8253(1)$ & $10173(1)$ & $7276(1)$ & $72(1)$ \\
\hline $\mathrm{O}(2)$ & $3885(1)$ & 2044(1) & $1465(1)$ & $70(1)$ \\
\hline $\mathrm{O}(3)$ & $7172(2)$ & $10960(1)$ & $1504(1)$ & $83(1)$ \\
\hline $\mathrm{O}(4)$ & $513(1)$ & $6754(1)$ & $5263(1)$ & $74(1)$ \\
\hline $\mathrm{O}(5)$ & $-81(2)$ & $6229(1)$ & $-1725(1)$ & $86(1)$ \\
\hline$C(1)$ & $7843(2)$ & $8436(2)$ & 4813(1) & $50(1)$ \\
\hline$C(2)$ & $8278(2)$ & $9458(2)$ & $5618(1)$ & $53(1)$ \\
\hline$C(3)$ & $7890(2)$ & $9242(2)$ & $6439(1)$ & $51(1)$ \\
\hline$C(4)$ & $7074(2)$ & $8009(2)$ & $6446(1)$ & $57(1)$ \\
\hline$C(5)$ & $6659(2)$ & 6998(2) & $5641(1)$ & $52(1)$ \\
\hline$C(6)$ & $7020(2)$ & 7191(1) & 4799(1) & $45(1)$ \\
\hline$C(7)$ & $6537(2)$ & $6107(1)$ & $3920(1)$ & $44(1)$ \\
\hline$C(8)$ & $6976(2)$ & $5052(2)$ & $3839(1)$ & $57(1)$ \\
\hline$C(9)$ & $6542(2)$ & $4058(2)$ & $3021(1)$ & $58(1)$ \\
\hline$C(10)$ & $5664(2)$ & $4068(1)$ & 2251(1) & $48(1)$ \\
\hline$C(11)$ & $5214(2)$ & $5127(1)$ & 2312(1) & $42(1)$ \\
\hline$C(12)$ & $5640(2)$ & $6138(1)$ & $3150(1)$ & $41(1)$ \\
\hline$C(13)$ & $4299(2)$ & $5199(1)$ & $1534(1)$ & $47(1)$ \\
\hline$C(14)$ & $3551(2)$ & $5335(1)$ & $920(1)$ & $50(1)$ \\
\hline$C(15)$ & $2635(2)$ & $5576(2)$ & $239(1)$ & $49(1)$ \\
\hline$C(16)$ & $2313(2)$ & $6688(2)$ & $448(1)$ & $66(1)$ \\
\hline$C(17)$ & $1414(2)$ & 6941(2) & $-185(1)$ & $69(1)$ \\
\hline$C(18)$ & $827(2)$ & $6077(2)$ & $-1049(1)$ & $60(1)$ \\
\hline$C(19)$ & $1166(2)$ & 4983(2) & $-1279(1)$ & 71(1) \\
\hline$C(20)$ & $2056(2)$ & $4724(2)$ & $-648(1)$ & $65(1)$ \\
\hline$C(21)$ & $5069(2)$ & $7216(1)$ & $3228(1)$ & $39(1)$ \\
\hline$C(22)$ & $5585(2)$ & $8163(1)$ & $2805(1)$ & $40(1)$ \\
\hline$C(23)$ & 4949(2) & $9138(1)$ & $2786(1)$ & $46(1)$ \\
\hline$C(24)$ & $5450(2)$ & $10046(2)$ & $2356(1)$ & $53(1)$ \\
\hline$C(25)$ & $6686(2)$ & $10117(2)$ & 1894(1) & $56(1)$ \\
\hline$C(26)$ & $7322(2)$ & $9144(2)$ & 1912(1) & $55(1)$ \\
\hline$C(27)$ & $6798(2)$ & $8225(1)$ & $2330(1)$ & $48(1)$ \\
\hline$C(28)$ & $3885(2)$ & $7148(1)$ & $3776(1)$ & $40(1)$ \\
\hline$C(29)$ & $4020(2)$ & $8103(1)$ & $4576(1)$ & $50(1)$ \\
\hline$C(30)$ & $2926(2)$ & $8008(2)$ & $5097(1)$ & $53(1)$ \\
\hline
\end{tabular}




$\begin{array}{lrrrr}\mathrm{C}(31) & 1663(2) & 6949(2) & 4809(1) & 50(1) \\ \mathrm{C}(32) & 1500(2) & 5991(2) & 4002(1) & 59(1) \\ \mathrm{C}(33) & 2605(2) & 6077(2) & 3501(1) & 52(1) \\ \mathrm{C}(34) & 9083(2) & 11446(2) & 7310(2) & 83(1) \\ \mathrm{C}(35) & 5174(2) & 2962(2) & 1379(1) & 59(1) \\ \mathrm{C}(36) & 3295(3) & 981(2) & 653(2) & 109(1) \\ \mathrm{C}(37) & 649(2) & 7661(2) & 6120(1) & 75(1) \\ \mathrm{C}(38) & -603(2) & 7256(2) & -1485(2) & 96(1)\end{array}$


Table S3. Bond lengths [A] and angles [deg] for y2038.

$\begin{array}{ll}\mathrm{O}(1)-\mathrm{C}(3) & 1.3657(19) \\ \mathrm{O}(1)-\mathrm{C}(34) & 1.406(2) \\ \mathrm{O}(2)-\mathrm{C}(35) & 1.4074(19) \\ \mathrm{O}(2)-\mathrm{C}(36) & 1.415(2) \\ \mathrm{O}(3)-\mathrm{C}(25) & 1.2342(18) \\ \mathrm{O}(4)-\mathrm{C}(31) & 1.3656(18) \\ \mathrm{O}(4)-\mathrm{C}(37) & 1.409(2) \\ \mathrm{O}(5)-\mathrm{C}(18) & 1.366(2) \\ \mathrm{O}(5)-\mathrm{C}(38) & 1.412(2) \\ \mathrm{C}(1)-\mathrm{C}(2) & 1.382(2) \\ \mathrm{C}(1)-\mathrm{C}(6) & 1.388(2) \\ \mathrm{C}(1)-\mathrm{H}(1) & 0.9300 \\ \mathrm{C}(2)-\mathrm{C}(3) & 1.377(2) \\ \mathrm{C}(2)-\mathrm{H}(2) & 0.9300 \\ \mathrm{C}(3)-\mathrm{C}(4) & 1.383(2) \\ \mathrm{C}(4)-\mathrm{C}(5) & 1.377(2) \\ \mathrm{C}(4)-\mathrm{H}(4) & 0.9300 \\ \mathrm{C}(5)-\mathrm{C}(6) & 1.392(2) \\ \mathrm{C}(5)-\mathrm{H}(5) & 0.9300 \\ \mathrm{C}(6)-\mathrm{C}(7) & 1.490(2) \\ \mathrm{C}(7)-\mathrm{C}(8) & 1.389(2) \\ \mathrm{C}(7)-\mathrm{C}(12) & 1.400(2) \\ \mathrm{C}(8)-\mathrm{C}(9) & 1.378(2) \\ \mathrm{C}(8)-\mathrm{H}(8) & 0.9300 \\ \mathrm{C}(9)-\mathrm{C}(10) & 1.378(2) \\ \mathrm{C}(9)-\mathrm{H}(9) & 0.9300 \\ \mathrm{C}(10)-\mathrm{C}(11) & 1.4018(19) \\ \mathrm{C}(10)-\mathrm{C}(35) & 1.496(2) \\ \mathrm{C}(11)-\mathrm{C}(12) & 1.408(2) \\ \mathrm{C}(11)-\mathrm{C}(13) & 1.439(2) \\ \mathrm{C}(12)-\mathrm{C}(21) & 1.4947(19) \\ \mathrm{C}(13)-\mathrm{C}(14) & 1.195(2) \\ \mathrm{C}(14)-\mathrm{C}(15) & 1.431(2) \\ \mathrm{C}(15)-\mathrm{C}(16) & 1.377(2) \\ \mathrm{C}(15)-\mathrm{C}(20) & 1.386(2) \\ \mathrm{C}(16)-\mathrm{C}(17) & 1.380(2) \\ \mathrm{C}(16)-\mathrm{H}(16) & 0.9300 \\ \mathrm{C}(17)-\mathrm{C}(18) & 1.367(2) \\ \mathrm{C}(17)-\mathrm{H}(17) & 0.9300 \\ \mathrm{C}(18)-\mathrm{C}(19) & \\ \mathrm{C}(19)-\mathrm{C}(20) & \\ & \\ & \\ & \end{array}$




\begin{tabular}{|c|c|}
\hline $\mathrm{C}(19)-\mathrm{H}(19)$ & 0.9300 \\
\hline $\mathrm{C}(20)-\mathrm{H}(20)$ & 0.9300 \\
\hline $\mathrm{C}(21)-\mathrm{C}(22)$ & $1.3659(19$ \\
\hline$C(21)-C(28)$ & $1.485(2)$ \\
\hline $\mathrm{C}(22)-\mathrm{C}(27)$ & $1.445(2)$ \\
\hline$C(22)-C(23)$ & $1.4461(19$ \\
\hline$C(23)-C(24)$ & $1.335(2)$ \\
\hline $\mathrm{C}(23)-\mathrm{H}(23)$ & 0.9300 \\
\hline$C(24)-C(25)$ & $1.452(2)$ \\
\hline $\mathrm{C}(24)-\mathrm{H}(24)$ & 0.9300 \\
\hline$C(25)-C(26)$ & $1.443(2)$ \\
\hline$C(26)-C(27)$ & $1.337(2)$ \\
\hline $\mathrm{C}(26)-\mathrm{H}(26)$ & 0.9300 \\
\hline $\mathrm{C}(27)-\mathrm{H}(27)$ & 0.9300 \\
\hline$C(28)-C(29)$ & $1.378(2)$ \\
\hline C(28)-C(33) & $1.391(2)$ \\
\hline$C(29)-C(30)$ & $1.386(2)$ \\
\hline $\mathrm{C}(29)-\mathrm{H}(29)$ & 0.9300 \\
\hline $\mathrm{C}(30)-\mathrm{C}(31)$ & $1.374(2)$ \\
\hline $\mathrm{C}(30)-\mathrm{H}(30)$ & 0.9300 \\
\hline $\mathrm{C}(31)-\mathrm{C}(32)$ & $1.380(2)$ \\
\hline$C(32)-C(33)$ & $1.375(2)$ \\
\hline $\mathrm{C}(32)-\mathrm{H}(32)$ & 0.9300 \\
\hline C(33)-H(33) & 0.9300 \\
\hline $\mathrm{C}(34)-\mathrm{H}(34 \mathrm{~A})$ & 0.9600 \\
\hline $\mathrm{C}(34)-\mathrm{H}(34 \mathrm{~B})$ & 0.9600 \\
\hline $\mathrm{C}(34)-\mathrm{H}(34 \mathrm{C})$ & 0.9600 \\
\hline $\mathrm{C}(35)-\mathrm{H}(35 \mathrm{~A})$ & 0.9700 \\
\hline $\mathrm{C}(35)-\mathrm{H}(35 \mathrm{~B})$ & 0.9700 \\
\hline $\mathrm{C}(36)-\mathrm{H}(36 \mathrm{~A})$ & 0.9600 \\
\hline $\mathrm{C}(36)-\mathrm{H}(36 \mathrm{~B})$ & 0.9600 \\
\hline $\mathrm{C}(36)-\mathrm{H}(36 \mathrm{C})$ & 0.9600 \\
\hline $\mathrm{C}(37)-\mathrm{H}(37 \mathrm{~A})$ & 0.9600 \\
\hline $\mathrm{C}(37)-\mathrm{H}(37 \mathrm{~B})$ & 0.9600 \\
\hline $\mathrm{C}(37)-\mathrm{H}(37 \mathrm{C})$ & 0.9600 \\
\hline $\mathrm{C}(38)-\mathrm{H}(38 \mathrm{~A})$ & 0.9600 \\
\hline $\mathrm{C}(38)-\mathrm{H}(38 \mathrm{~B})$ & 0.9600 \\
\hline $\mathrm{C}(38)-\mathrm{H}(38 \mathrm{C})$ & 0.9600 \\
\hline $\mathrm{C}(3)-\mathrm{O}(1)-\mathrm{C}(34)$ & $118.24(15)$ \\
\hline $\mathrm{C}(35)-\mathrm{O}(2)-\mathrm{C}(36)$ & $112.31(15)$ \\
\hline $\mathrm{C}(31)-\mathrm{O}(4)-\mathrm{C}(37)$ & $118.25(14)$ \\
\hline $\mathrm{C}(18)-\mathrm{O}(5)-\mathrm{C}(38)$ & $118.34(16)$ \\
\hline $\mathrm{C}(2)-\mathrm{C}(1)-\mathrm{C}(6)$ & $122.19(15)$ \\
\hline
\end{tabular}




\begin{tabular}{|c|c|}
\hline $\mathrm{C}(2)-\mathrm{C}(1)-\mathrm{H}(1)$ & 118.9 \\
\hline $\mathrm{C}(6)-\mathrm{C}(1)-\mathrm{H}(1)$ & 118.9 \\
\hline $\mathrm{C}(3)-\mathrm{C}(2)-\mathrm{C}(1)$ & $119.39(15)$ \\
\hline $\mathrm{C}(3)-\mathrm{C}(2)-\mathrm{H}(2)$ & 120.3 \\
\hline $\mathrm{C}(1)-\mathrm{C}(2)-\mathrm{H}(2)$ & 120.3 \\
\hline $\mathrm{O}(1)-\mathrm{C}(3)-\mathrm{C}(2)$ & $124.60(16)$ \\
\hline $\mathrm{O}(1)-\mathrm{C}(3)-\mathrm{C}(4)$ & $115.80(15)$ \\
\hline$C(2)-C(3)-C(4)$ & $119.60(15)$ \\
\hline$C(5)-C(4)-C(3)$ & $120.46(15)$ \\
\hline $\mathrm{C}(5)-\mathrm{C}(4)-\mathrm{H}(4)$ & 119.8 \\
\hline $\mathrm{C}(3)-\mathrm{C}(4)-\mathrm{H}(4)$ & 119.8 \\
\hline$C(4)-C(5)-C(6)$ & $121.14(15)$ \\
\hline $\mathrm{C}(4)-\mathrm{C}(5)-\mathrm{H}(5)$ & 119.4 \\
\hline $\mathrm{C}(6)-\mathrm{C}(5)-\mathrm{H}(5)$ & 119.4 \\
\hline$C(1)-C(6)-C(5)$ & $117.21(15)$ \\
\hline$C(1)-C(6)-C(7)$ & $121.40(14)$ \\
\hline$C(5)-C(6)-C(7)$ & $121.39(14)$ \\
\hline$C(8)-C(7)-C(12)$ & $118.37(14)$ \\
\hline $\mathrm{C}(8)-\mathrm{C}(7)-\mathrm{C}(6)$ & $121.05(14)$ \\
\hline$C(12)-C(7)-C(6)$ & $120.57(13)$ \\
\hline $\mathrm{C}(9)-\mathrm{C}(8)-\mathrm{C}(7)$ & $120.91(15)$ \\
\hline $\mathrm{C}(9)-\mathrm{C}(8)-\mathrm{H}(8)$ & 119.5 \\
\hline $\mathrm{C}(7)-\mathrm{C}(8)-\mathrm{H}(8)$ & 119.5 \\
\hline$C(10)-C(9)-C(8)$ & $121.86(14)$ \\
\hline $\mathrm{C}(10)-\mathrm{C}(9)-\mathrm{H}(9)$ & 119.1 \\
\hline $\mathrm{C}(8)-\mathrm{C}(9)-\mathrm{H}(9)$ & 119.1 \\
\hline $\mathrm{C}(9)-\mathrm{C}(10)-\mathrm{C}(11)$ & $118.37(14)$ \\
\hline$C(9)-C(10)-C(35)$ & $120.91(14)$ \\
\hline $\mathrm{C}(11)-\mathrm{C}(10)-\mathrm{C}(35)$ & $120.71(14)$ \\
\hline$C(10)-C(11)-C(12)$ & $120.08(14)$ \\
\hline$C(10)-C(11)-C(13)$ & $121.23(14)$ \\
\hline$C(12)-C(11)-C(13)$ & $118.69(13)$ \\
\hline$C(7)-C(12)-C(11)$ & $120.41(13)$ \\
\hline$C(7)-C(12)-C(21)$ & $119.96(13)$ \\
\hline$C(11)-C(12)-C(21)$ & $119.56(13)$ \\
\hline$C(14)-C(13)-C(11)$ & $175.94(16)$ \\
\hline $\mathrm{C}(13)-\mathrm{C}(14)-\mathrm{C}(15)$ & $175.40(17)$ \\
\hline$C(16)-C(15)-C(20)$ & $117.65(16)$ \\
\hline$C(16)-C(15)-C(14)$ & $120.32(14)$ \\
\hline$C(20)-C(15)-C(14)$ & $122.03(16)$ \\
\hline$C(15)-C(16)-C(17)$ & $121.89(16)$ \\
\hline $\mathrm{C}(15)-\mathrm{C}(16)-\mathrm{H}(16)$ & 119.1 \\
\hline $\mathrm{C}(17)-\mathrm{C}(16)-\mathrm{H}(16)$ & 119.1 \\
\hline$C(18)-C(17)-C(16)$ & $119.63(18)$ \\
\hline
\end{tabular}




\begin{tabular}{|c|c|}
\hline $\mathrm{C}(18)-\mathrm{C}(17)-\mathrm{H}(17)$ & 120.2 \\
\hline $\mathrm{C}(16)-\mathrm{C}(17)-\mathrm{H}(17)$ & 120.2 \\
\hline $\mathrm{O}(5)-\mathrm{C}(18)-\mathrm{C}(17)$ & $124.41(18)$ \\
\hline $\mathrm{O}(5)-\mathrm{C}(18)-\mathrm{C}(19)$ & $116.25(17)$ \\
\hline $\mathrm{C}(17)-\mathrm{C}(18)-\mathrm{C}(19)$ & $119.34(17)$ \\
\hline$C(18)-C(19)-C(20)$ & $121.14(17)$ \\
\hline $\mathrm{C}(18)-\mathrm{C}(19)-\mathrm{H}(19)$ & 119.4 \\
\hline $\mathrm{C}(20)-\mathrm{C}(19)-\mathrm{H}(19)$ & 119.4 \\
\hline$C(19)-C(20)-C(15)$ & $120.31(17)$ \\
\hline $\mathrm{C}(19)-\mathrm{C}(20)-\mathrm{H}(20)$ & 119.8 \\
\hline $\mathrm{C}(15)-\mathrm{C}(20)-\mathrm{H}(20)$ & 119.8 \\
\hline$C(22)-C(21)-C(28)$ & $123.49(12)$ \\
\hline$C(22)-C(21)-C(12)$ & $121.78(13)$ \\
\hline$C(28)-C(21)-C(12)$ & $114.72(12)$ \\
\hline $\mathrm{C}(21)-\mathrm{C}(22)-\mathrm{C}(27)$ & $121.03(13)$ \\
\hline$C(21)-C(22)-C(23)$ & $122.91(13)$ \\
\hline$C(27)-C(22)-C(23)$ & $116.05(13)$ \\
\hline $\mathrm{C}(24)-\mathrm{C}(23)-\mathrm{C}(22)$ & $122.25(15)$ \\
\hline $\mathrm{C}(24)-\mathrm{C}(23)-\mathrm{H}(23)$ & 118.9 \\
\hline $\mathrm{C}(22)-\mathrm{C}(23)-\mathrm{H}(23)$ & 118.9 \\
\hline $\mathrm{C}(23)-\mathrm{C}(24)-\mathrm{C}(25)$ & $121.43(15)$ \\
\hline $\mathrm{C}(23)-\mathrm{C}(24)-\mathrm{H}(24)$ & 119.3 \\
\hline $\mathrm{C}(25)-\mathrm{C}(24)-\mathrm{H}(24)$ & 119.3 \\
\hline $\mathrm{O}(3)-\mathrm{C}(25)-\mathrm{C}(26)$ & $121.47(17)$ \\
\hline $\mathrm{O}(3)-\mathrm{C}(25)-\mathrm{C}(24)$ & $122.11(16)$ \\
\hline$C(26)-C(25)-C(24)$ & $116.42(14)$ \\
\hline$C(27)-C(26)-C(25)$ & $121.77(16)$ \\
\hline $\mathrm{C}(27)-\mathrm{C}(26)-\mathrm{H}(26)$ & 119.1 \\
\hline $\mathrm{C}(25)-\mathrm{C}(26)-\mathrm{H}(26)$ & 119.1 \\
\hline$C(26)-C(27)-C(22)$ & $122.05(14)$ \\
\hline $\mathrm{C}(26)-\mathrm{C}(27)-\mathrm{H}(27)$ & 119.0 \\
\hline $\mathrm{C}(22)-\mathrm{C}(27)-\mathrm{H}(27)$ & 119.0 \\
\hline $\mathrm{C}(29)-\mathrm{C}(28)-\mathrm{C}(33)$ & $117.73(14)$ \\
\hline $\mathrm{C}(29)-\mathrm{C}(28)-\mathrm{C}(21)$ & $122.19(13)$ \\
\hline$C(33)-C(28)-C(21)$ & $120.05(13)$ \\
\hline $\mathrm{C}(28)-\mathrm{C}(29)-\mathrm{C}(30)$ & $121.64(15)$ \\
\hline $\mathrm{C}(28)-\mathrm{C}(29)-\mathrm{H}(29)$ & 119.2 \\
\hline $\mathrm{C}(30)-\mathrm{C}(29)-\mathrm{H}(29)$ & 119.2 \\
\hline $\mathrm{C}(31)-\mathrm{C}(30)-\mathrm{C}(29)$ & $119.73(15)$ \\
\hline $\mathrm{C}(31)-\mathrm{C}(30)-\mathrm{H}(30)$ & 120.1 \\
\hline $\mathrm{C}(29)-\mathrm{C}(30)-\mathrm{H}(30)$ & 120.1 \\
\hline $\mathrm{O}(4)-\mathrm{C}(31)-\mathrm{C}(30)$ & $124.88(14)$ \\
\hline $\mathrm{O}(4)-\mathrm{C}(31)-\mathrm{C}(32)$ & $115.67(15)$ \\
\hline $\mathrm{C}(30)-\mathrm{C}(31)-\mathrm{C}(32)$ & $119.45(15)$ \\
\hline
\end{tabular}




\begin{tabular}{|c|c|}
\hline $\mathrm{C}(33)-\mathrm{C}(32)-\mathrm{C}(31)$ & $120.46(15)$ \\
\hline $\mathrm{C}(33)-\mathrm{C}(32)-\mathrm{H}(32)$ & 119.8 \\
\hline $\mathrm{C}(31)-\mathrm{C}(32)-\mathrm{H}(32)$ & 119.8 \\
\hline $\mathrm{C}(32)-\mathrm{C}(33)-\mathrm{C}(28)$ & $120.95(14)$ \\
\hline $\mathrm{C}(32)-\mathrm{C}(33)-\mathrm{H}(33)$ & 119.5 \\
\hline $\mathrm{C}(28)-\mathrm{C}(33)-\mathrm{H}(33)$ & 119.5 \\
\hline $\mathrm{O}(1)-\mathrm{C}(34)-\mathrm{H}(34 \mathrm{~A})$ & 109.5 \\
\hline $\mathrm{O}(1)-\mathrm{C}(34)-\mathrm{H}(34 \mathrm{~B})$ & 109.5 \\
\hline $\mathrm{H}(34 \mathrm{~A})-\mathrm{C}(34)-\mathrm{H}(34 \mathrm{~B})$ & 109.5 \\
\hline $\mathrm{O}(1)-\mathrm{C}(34)-\mathrm{H}(34 \mathrm{C})$ & 109.5 \\
\hline $\mathrm{H}(34 \mathrm{~A})-\mathrm{C}(34)-\mathrm{H}(34 \mathrm{C})$ & 109.5 \\
\hline $\mathrm{H}(34 \mathrm{~B})-\mathrm{C}(34)-\mathrm{H}(34 \mathrm{C})$ & 109.5 \\
\hline $\mathrm{O}(2)-\mathrm{C}(35)-\mathrm{C}(10)$ & $107.97(13)$ \\
\hline $\mathrm{O}(2)-\mathrm{C}(35)-\mathrm{H}(35 \mathrm{~A})$ & 110.1 \\
\hline $\mathrm{C}(10)-\mathrm{C}(35)-\mathrm{H}(35 \mathrm{~A})$ & 110.1 \\
\hline $\mathrm{O}(2)-\mathrm{C}(35)-\mathrm{H}(35 \mathrm{~B})$ & 110.1 \\
\hline $\mathrm{C}(10)-\mathrm{C}(35)-\mathrm{H}(35 \mathrm{~B})$ & 110.1 \\
\hline $\mathrm{H}(35 \mathrm{~A})-\mathrm{C}(35)-\mathrm{H}(35 \mathrm{~B})$ & 108.4 \\
\hline $\mathrm{O}(2)-\mathrm{C}(36)-\mathrm{H}(36 \mathrm{~A})$ & 109.5 \\
\hline $\mathrm{O}(2)-\mathrm{C}(36)-\mathrm{H}(36 \mathrm{~B})$ & 109.5 \\
\hline $\mathrm{H}(36 \mathrm{~A})-\mathrm{C}(36)-\mathrm{H}(36 \mathrm{~B})$ & 109.5 \\
\hline $\mathrm{O}(2)-\mathrm{C}(36)-\mathrm{H}(36 \mathrm{C})$ & 109.5 \\
\hline $\mathrm{H}(36 \mathrm{~A})-\mathrm{C}(36)-\mathrm{H}(36 \mathrm{C})$ & 109.5 \\
\hline $\mathrm{H}(36 \mathrm{~B})-\mathrm{C}(36)-\mathrm{H}(36 \mathrm{C})$ & 109.5 \\
\hline $\mathrm{O}(4)-\mathrm{C}(37)-\mathrm{H}(37 \mathrm{~A})$ & 109.5 \\
\hline $\mathrm{O}(4)-\mathrm{C}(37)-\mathrm{H}(37 \mathrm{~B})$ & 109.5 \\
\hline $\mathrm{H}(37 \mathrm{~A})-\mathrm{C}(37)-\mathrm{H}(37 \mathrm{~B})$ & 109.5 \\
\hline $\mathrm{O}(4)-\mathrm{C}(37)-\mathrm{H}(37 \mathrm{C})$ & 109.5 \\
\hline $\mathrm{H}(37 \mathrm{~A})-\mathrm{C}(37)-\mathrm{H}(37 \mathrm{C})$ & 109.5 \\
\hline $\mathrm{H}(37 \mathrm{~B})-\mathrm{C}(37)-\mathrm{H}(37 \mathrm{C})$ & 109.5 \\
\hline $\mathrm{O}(5)-\mathrm{C}(38)-\mathrm{H}(38 \mathrm{~A})$ & 109.5 \\
\hline $\mathrm{O}(5)-\mathrm{C}(38)-\mathrm{H}(38 \mathrm{~B})$ & 109.5 \\
\hline $\mathrm{H}(38 \mathrm{~A})-\mathrm{C}(38)-\mathrm{H}(38 \mathrm{~B})$ & 109.5 \\
\hline $\mathrm{O}(5)-\mathrm{C}(38)-\mathrm{H}(38 \mathrm{C})$ & 109.5 \\
\hline $\mathrm{H}(38 \mathrm{~A})-\mathrm{C}(38)-\mathrm{H}(38 \mathrm{C})$ & 109.5 \\
\hline $\mathrm{H}(38 \mathrm{~B})-\mathrm{C}(38)-\mathrm{H}(38 \mathrm{C})$ & 109.5 \\
\hline
\end{tabular}


Table S4. Anisotropic displacement parameters $(A \wedge 2 \times 10 \wedge 3)$ for $y 2038$.

The anisotropic displacement factor exponent takes the form:

$$
-2 \mathrm{pi}^{\wedge} 2\left[\mathrm{~h}^{\wedge} 2 \mathrm{a}^{* \wedge} 2 \mathrm{U} 11+\ldots+2 \mathrm{hk} \mathrm{a} \mathrm{b}^{*} \mathrm{U} 12\right]
$$

\begin{tabular}{|c|c|c|c|c|c|c|}
\hline & U11 & U22 & U33 & U23 & U13 & U12 \\
\hline $\mathrm{O}(1)$ & $79(1)$ & $66(1)$ & $65(1)$ & $4(1)$ & 11(1) & $28(1)$ \\
\hline $\mathrm{O}(2)$ & $82(1)$ & $58(1)$ & $55(1)$ & $5(1)$ & $13(1)$ & $13(1)$ \\
\hline $\mathrm{O}(3)$ & $97(1)$ & $71(1)$ & 103(1) & $57(1)$ & $34(1)$ & $31(1)$ \\
\hline $\mathrm{O}(4)$ & $58(1)$ & $88(1)$ & $70(1)$ & $11(1)$ & $28(1)$ & $22(1)$ \\
\hline $\mathrm{O}(5)$ & $75(1)$ & $95(1)$ & $78(1)$ & $29(1)$ & $-21(1)$ & $23(1)$ \\
\hline $\mathrm{C}(1)$ & $47(1)$ & $57(1)$ & $48(1)$ & $22(1)$ & $6(1)$ & $17(1)$ \\
\hline$C(2)$ & $48(1)$ & $49(1)$ & $61(1)$ & $18(1)$ & $3(1)$ & $14(1)$ \\
\hline $\mathrm{C}(3)$ & $46(1)$ & $57(1)$ & $52(1)$ & $10(1)$ & $4(1)$ & $26(1)$ \\
\hline$C(4)$ & $56(1)$ & $69(1)$ & $52(1)$ & $24(1)$ & $16(1)$ & $26(1)$ \\
\hline$C(5)$ & $50(1)$ & $52(1)$ & $58(1)$ & $23(1)$ & $12(1)$ & $18(1)$ \\
\hline$C(6)$ & $39(1)$ & $50(1)$ & $50(1)$ & $19(1)$ & $5(1)$ & $19(1)$ \\
\hline$C(7)$ & $42(1)$ & $44(1)$ & $51(1)$ & $21(1)$ & $10(1)$ & $17(1)$ \\
\hline $\mathrm{C}(8)$ & $59(1)$ & $57(1)$ & $65(1)$ & $28(1)$ & $6(1)$ & $30(1)$ \\
\hline$C(9)$ & $67(1)$ & $50(1)$ & $73(1)$ & $26(1)$ & 19(1) & $36(1)$ \\
\hline$C(10)$ & $56(1)$ & $44(1)$ & $54(1)$ & $20(1)$ & $22(1)$ & $24(1)$ \\
\hline$C(11)$ & $44(1)$ & $41(1)$ & $47(1)$ & $18(1)$ & $14(1)$ & $17(1)$ \\
\hline$C(12)$ & $41(1)$ & $39(1)$ & $49(1)$ & $18(1)$ & $13(1)$ & $17(1)$ \\
\hline $\mathrm{C}(13)$ & $54(1)$ & $42(1)$ & $48(1)$ & $13(1)$ & $14(1)$ & $21(1)$ \\
\hline$C(14)$ & $54(1)$ & $48(1)$ & $48(1)$ & $14(1)$ & $12(1)$ & $17(1)$ \\
\hline$C(15)$ & $48(1)$ & $53(1)$ & $42(1)$ & $15(1)$ & $7(1)$ & $13(1)$ \\
\hline$C(16)$ & $75(1)$ & $74(1)$ & $46(1)$ & $4(1)$ & $-3(1)$ & $35(1)$ \\
\hline$C(17)$ & $74(1)$ & $72(1)$ & $62(1)$ & $14(1)$ & $2(1)$ & $36(1)$ \\
\hline $\mathrm{C}(18)$ & $50(1)$ & $66(1)$ & $55(1)$ & $22(1)$ & $-4(1)$ & $8(1)$ \\
\hline$C(19)$ & $80(1)$ & $63(1)$ & $51(1)$ & $8(1)$ & $-10(1)$ & $12(1)$ \\
\hline $\mathrm{C}(20)$ & $79(1)$ & $54(1)$ & $55(1)$ & $11(1)$ & $3(1)$ & $19(1)$ \\
\hline$C(21)$ & $37(1)$ & $39(1)$ & $40(1)$ & $9(1)$ & $2(1)$ & $15(1)$ \\
\hline$C(22)$ & $39(1)$ & $38(1)$ & $44(1)$ & $12(1)$ & $4(1)$ & $15(1)$ \\
\hline$C(23)$ & $45(1)$ & $43(1)$ & $52(1)$ & $14(1)$ & $6(1)$ & $19(1)$ \\
\hline$C(24)$ & $60(1)$ & $43(1)$ & $61(1)$ & $21(1)$ & $5(1)$ & $24(1)$ \\
\hline$C(25)$ & $64(1)$ & $48(1)$ & $58(1)$ & $24(1)$ & $7(1)$ & $16(1)$ \\
\hline$C(26)$ & $56(1)$ & $58(1)$ & $61(1)$ & $28(1)$ & $20(1)$ & $25(1)$ \\
\hline$C(27)$ & $50(1)$ & $49(1)$ & $54(1)$ & $20(1)$ & $12(1)$ & $25(1)$ \\
\hline $\mathrm{C}(28)$ & $42(1)$ & $39(1)$ & $45(1)$ & $14(1)$ & $6(1)$ & $18(1)$ \\
\hline$C(29)$ & $43(1)$ & $43(1)$ & $58(1)$ & $5(1)$ & $7(1)$ & $14(1)$ \\
\hline$C(30)$ & $53(1)$ & $55(1)$ & $48(1)$ & $3(1)$ & $9(1)$ & $23(1)$ \\
\hline
\end{tabular}




$\begin{array}{lrrrrrr}\mathrm{C}(31) & 47(1) & 58(1) & 50(1) & 17(1) & 13(1) & 22(1) \\ \mathrm{C}(32) & 48(1) & 53(1) & 62(1) & 7(1) & 14(1) & 5(1) \\ \mathrm{C}(33) & 51(1) & 45(1) & 51(1) & 4(1) & 11(1) & 12(1) \\ \mathrm{C}(34) & 71(1) & 72(1) & 88(2) & -2(1) & 2(1) & 23(1) \\ \mathrm{C}(35) & 80(1) & 52(1) & 59(1) & 21(1) & 29(1) & 34(1) \\ \mathrm{C}(36) & 131(2) & 87(2) & 71(1) & -10(1) & -9(1) & 20(2) \\ \mathrm{C}(37) & 68(1) & 108(2) & 62(1) & 21(1) & 24(1) & 48(1) \\ \mathrm{C}(38) & 70(1) & 116(2) & 115(2) & 61(2) & 0(1) & 35(1)\end{array}$


Table S5. Hydrogen coordinates $(x 10 \wedge 4)$ and isotropic displacement parameters $(A \wedge 2 x$ $\left.10^{\wedge} 3\right)$ for y2038.

\begin{tabular}{|c|c|c|c|c|}
\hline & $\mathrm{x}$ & $\mathrm{y}$ & $\mathrm{z}$ & $\mathrm{U}(\mathrm{eq})$ \\
\hline $\mathrm{H}(1)$ & 8112 & 8589 & 4261 & 60 \\
\hline $\mathrm{H}(2)$ & 8826 & 10283 & 5606 & 64 \\
\hline $\mathrm{H}(4)$ & 6803 & 7862 & 6998 & 68 \\
\hline $\mathrm{H}(5)$ & 6128 & 6171 & 5660 & 63 \\
\hline $\mathrm{H}(8)$ & 7572 & 5015 & 4344 & 68 \\
\hline $\mathrm{H}(9)$ & 6849 & 3362 & 2987 & 69 \\
\hline $\mathrm{H}(16)$ & 2714 & 7284 & 1033 & 79 \\
\hline $\mathrm{H}(17)$ & 1209 & 7697 & -24 & 83 \\
\hline $\mathrm{H}(19)$ & 789 & 4405 & -1873 & 86 \\
\hline $\mathrm{H}(20)$ & 2270 & 3974 & -816 & 78 \\
\hline $\mathrm{H}(23)$ & 4165 & 9133 & 3083 & 55 \\
\hline $\mathrm{H}(24)$ & 4996 & 10645 & 2352 & 63 \\
\hline $\mathrm{H}(26)$ & 8117 & 9157 & 1624 & 66 \\
\hline $\mathrm{H}(27)$ & 7228 & 7607 & 2314 & 58 \\
\hline $\mathrm{H}(29)$ & 4865 & 8829 & 4770 & 61 \\
\hline $\mathrm{H}(30)$ & 3047 & 8659 & 5640 & 64 \\
\hline $\mathrm{H}(32)$ & 637 & 5283 & 3795 & 71 \\
\hline $\mathrm{H}(33)$ & 2494 & 5409 & 2972 & 62 \\
\hline $\mathrm{H}(34 \mathrm{~A})$ & 9988 & 11467 & 7118 & 100 \\
\hline $\mathrm{H}(34 \mathrm{~B})$ & 9273 & 11994 & 7937 & 100 \\
\hline $\mathrm{H}(34 \mathrm{C})$ & 8556 & 11745 & 6897 & 100 \\
\hline $\mathrm{H}(35 \mathrm{~A})$ & 4997 & 3260 & 839 & 71 \\
\hline $\mathrm{H}(35 \mathrm{~B})$ & 5918 & 2582 & 1297 & 71 \\
\hline $\mathrm{H}(36 \mathrm{~A})$ & 3031 & 1273 & 134 & 131 \\
\hline $\mathrm{H}(36 \mathrm{~B})$ & 2448 & 361 & 754 & 131 \\
\hline $\mathrm{H}(36 \mathrm{C})$ & 4006 & 588 & 518 & 131 \\
\hline $\mathrm{H}(37 \mathrm{~A})$ & 1426 & 7677 & 6566 & 90 \\
\hline $\mathrm{H}(37 \mathrm{~B})$ & -244 & 7424 & 6349 & 90 \\
\hline $\mathrm{H}(37 \mathrm{C})$ & 859 & 8501 & 6027 & 90 \\
\hline $\mathrm{H}(38 \mathrm{~A})$ & -1081 & 7194 & -962 & 116 \\
\hline $\mathrm{H}(38 \mathrm{~B})$ & -1280 & 7211 & -2009 & 116 \\
\hline $\mathrm{H}(38 \mathrm{C})$ & 197 & 8062 & -1323 & 116 \\
\hline
\end{tabular}


The HR-ESIMS spectrum of compound 1a

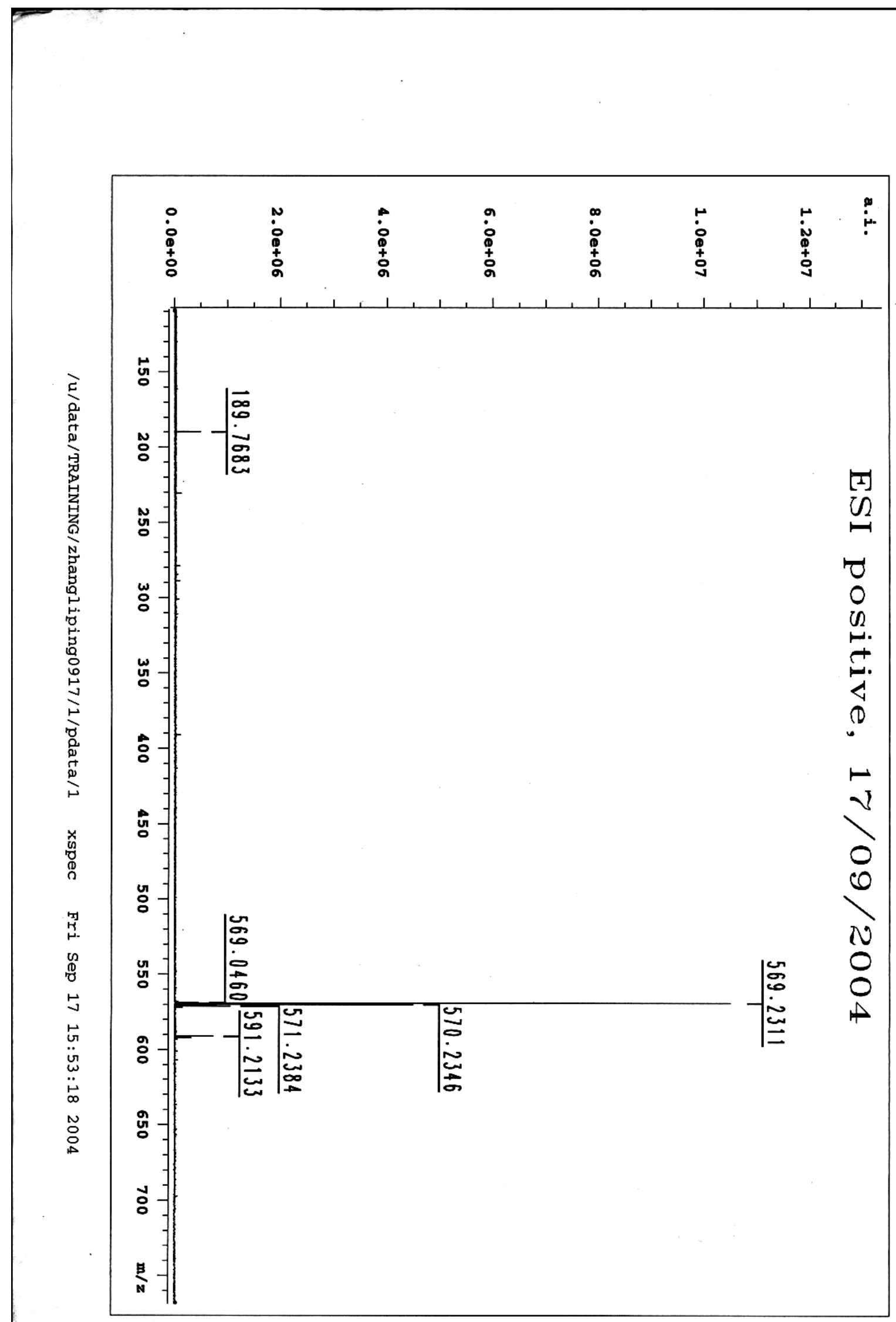


The HR-ESIMS spectrum of compound $\mathbf{1}$

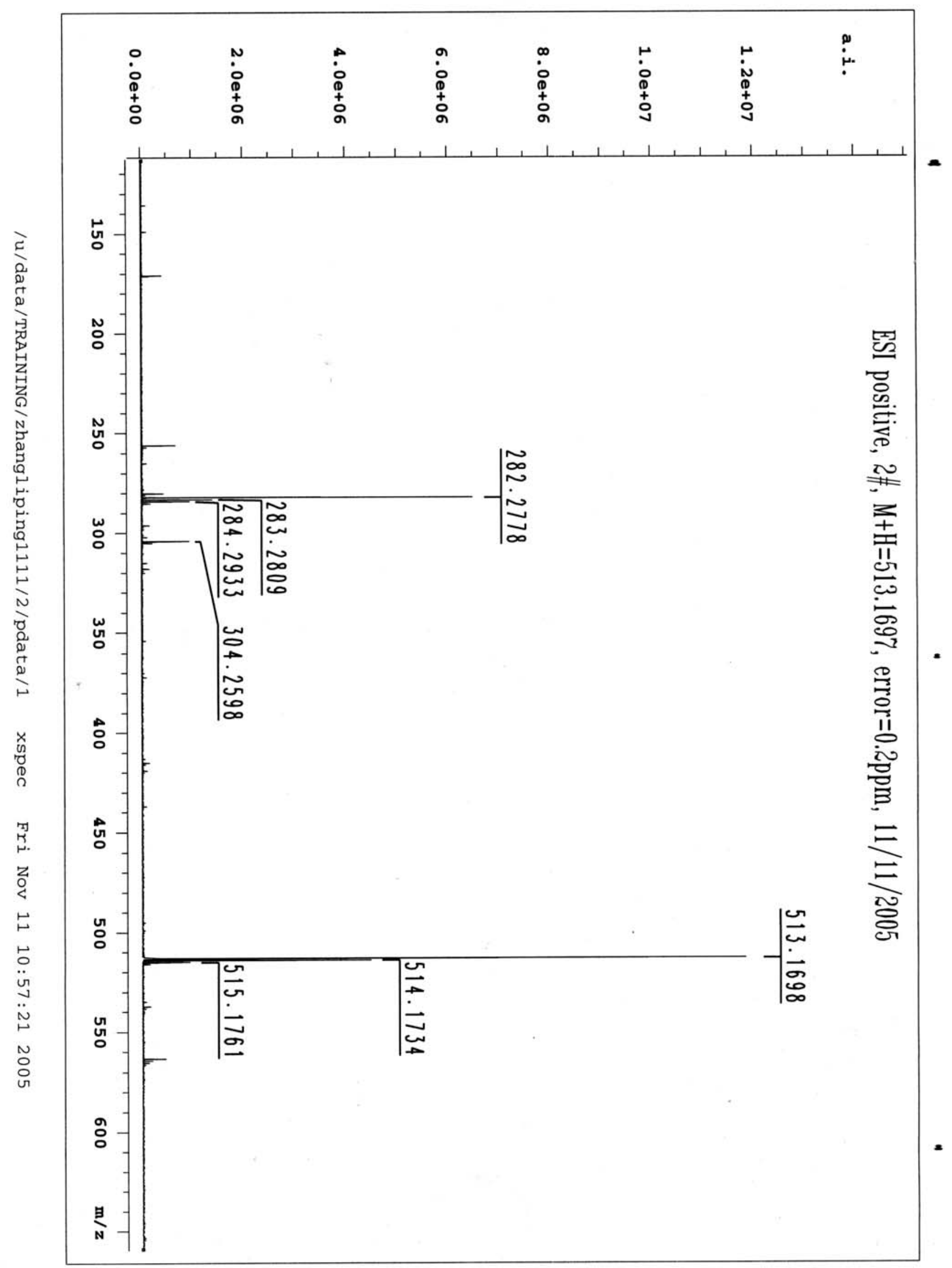


${ }^{1} \mathrm{H}$ NMR spectrum of compound 1a $\left(400 \mathrm{MHz}, \mathrm{CDCl}_{3}\right)$

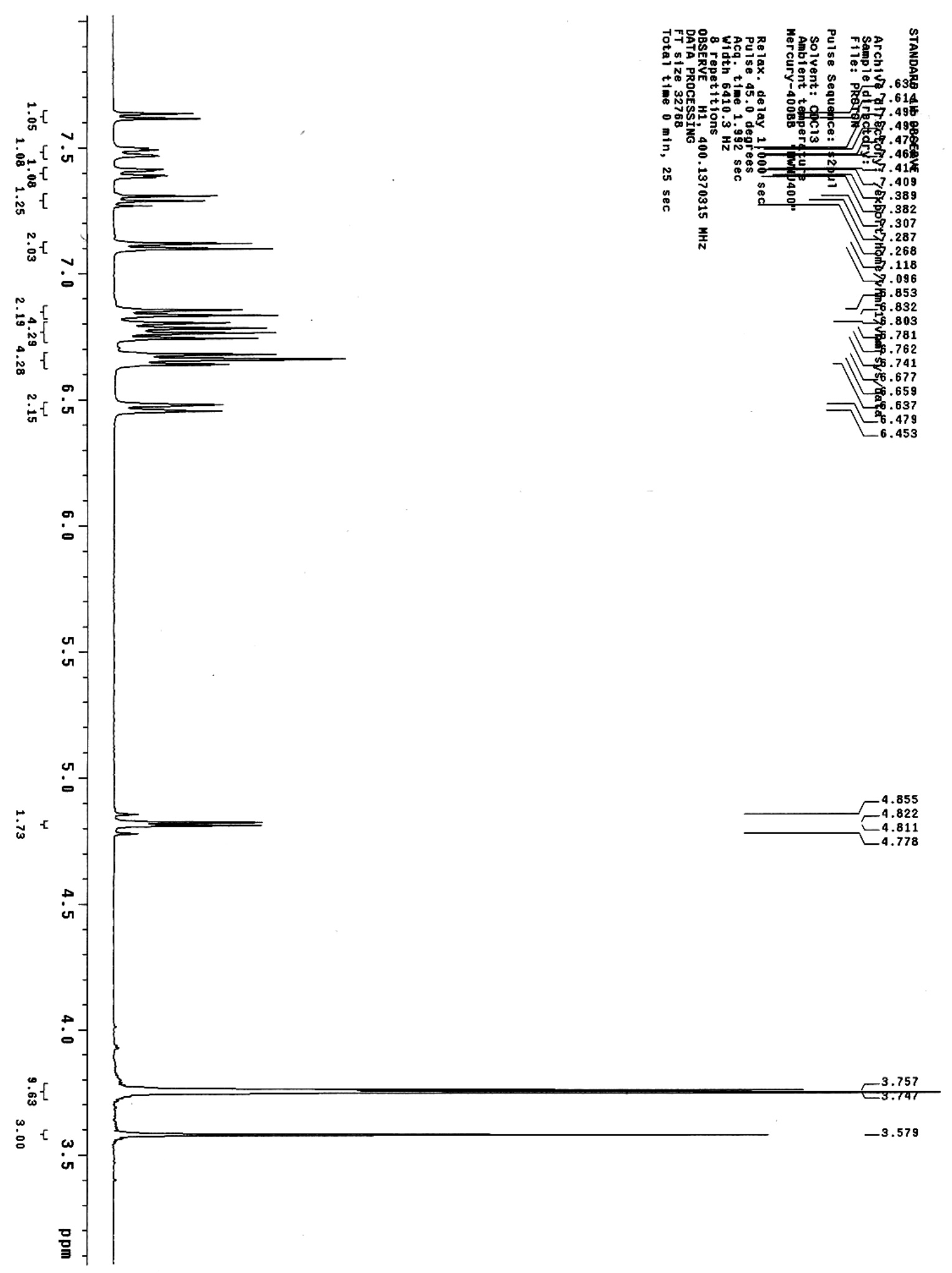


${ }^{13} \mathrm{C}$ NMR spectrum of compound $1 \mathbf{a}\left(400 \mathrm{MHz}, \mathrm{CDCl}_{3}\right)$

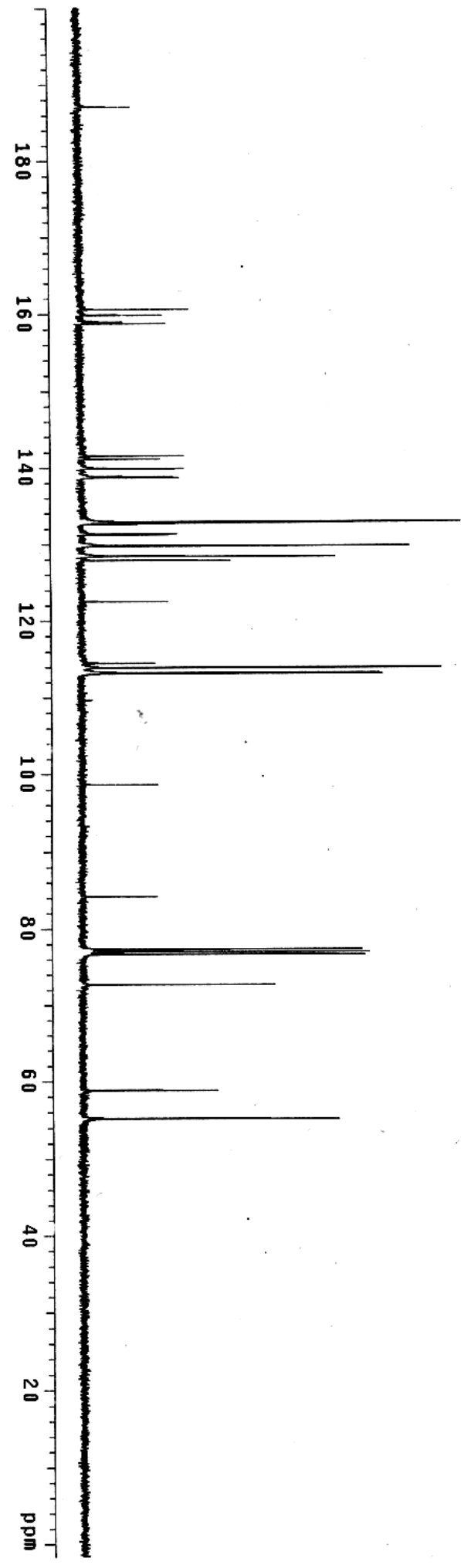

187.038

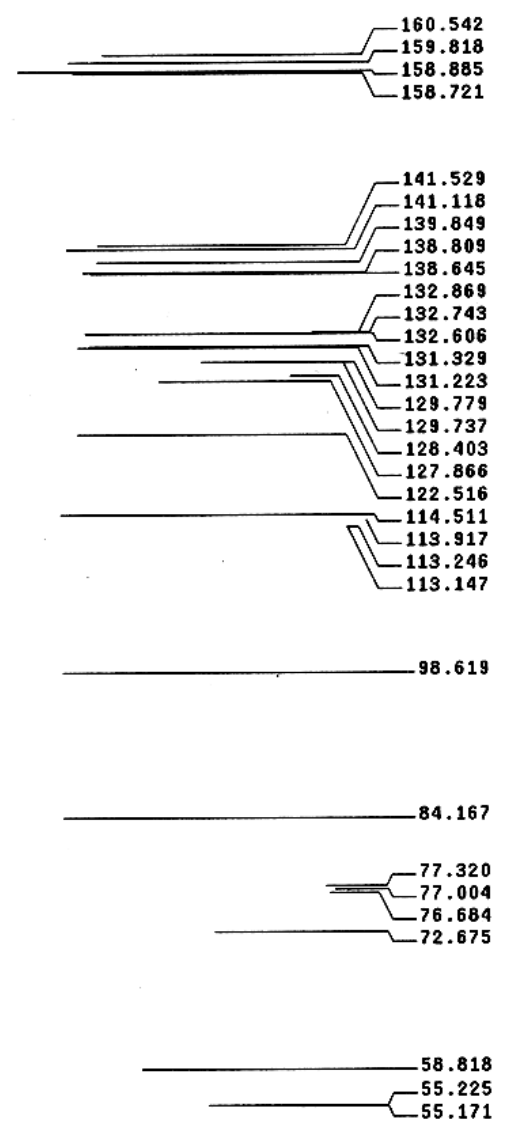




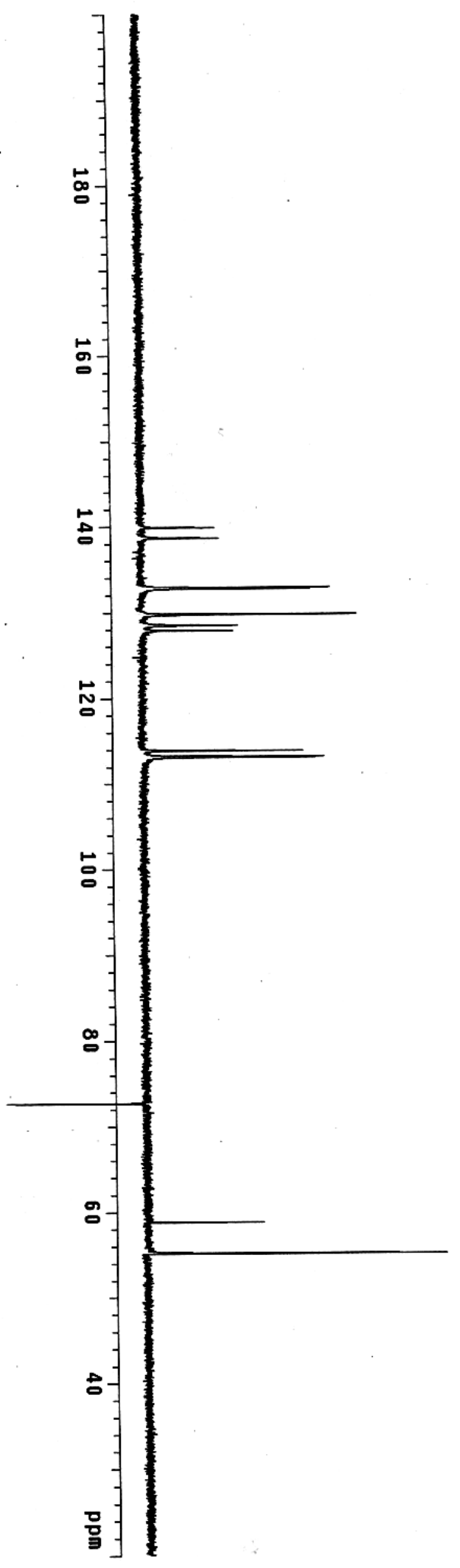


gCOSY spectrum of compound 1a $\left(400 \mathrm{MHz}, \mathrm{CDCl}_{3}\right)$

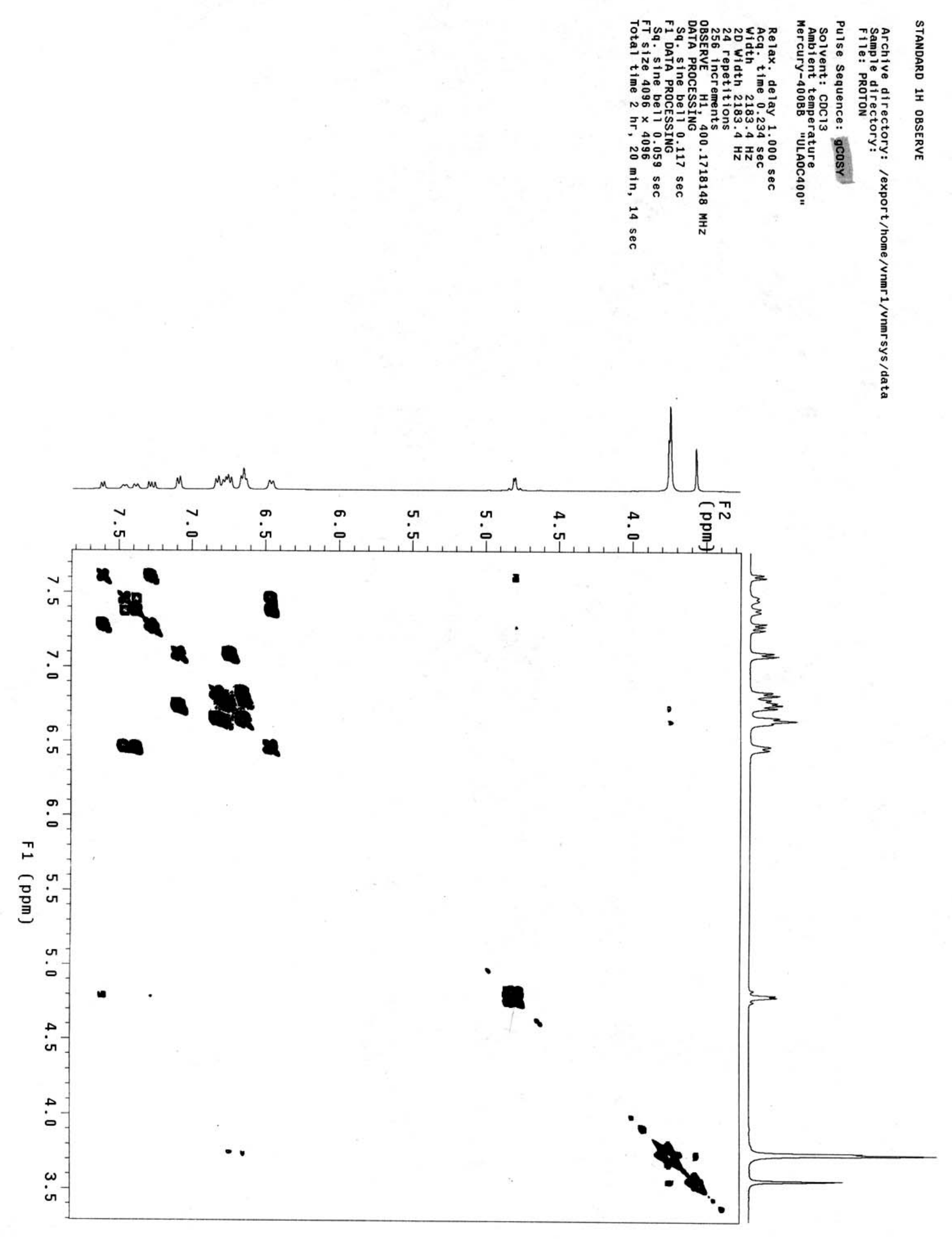




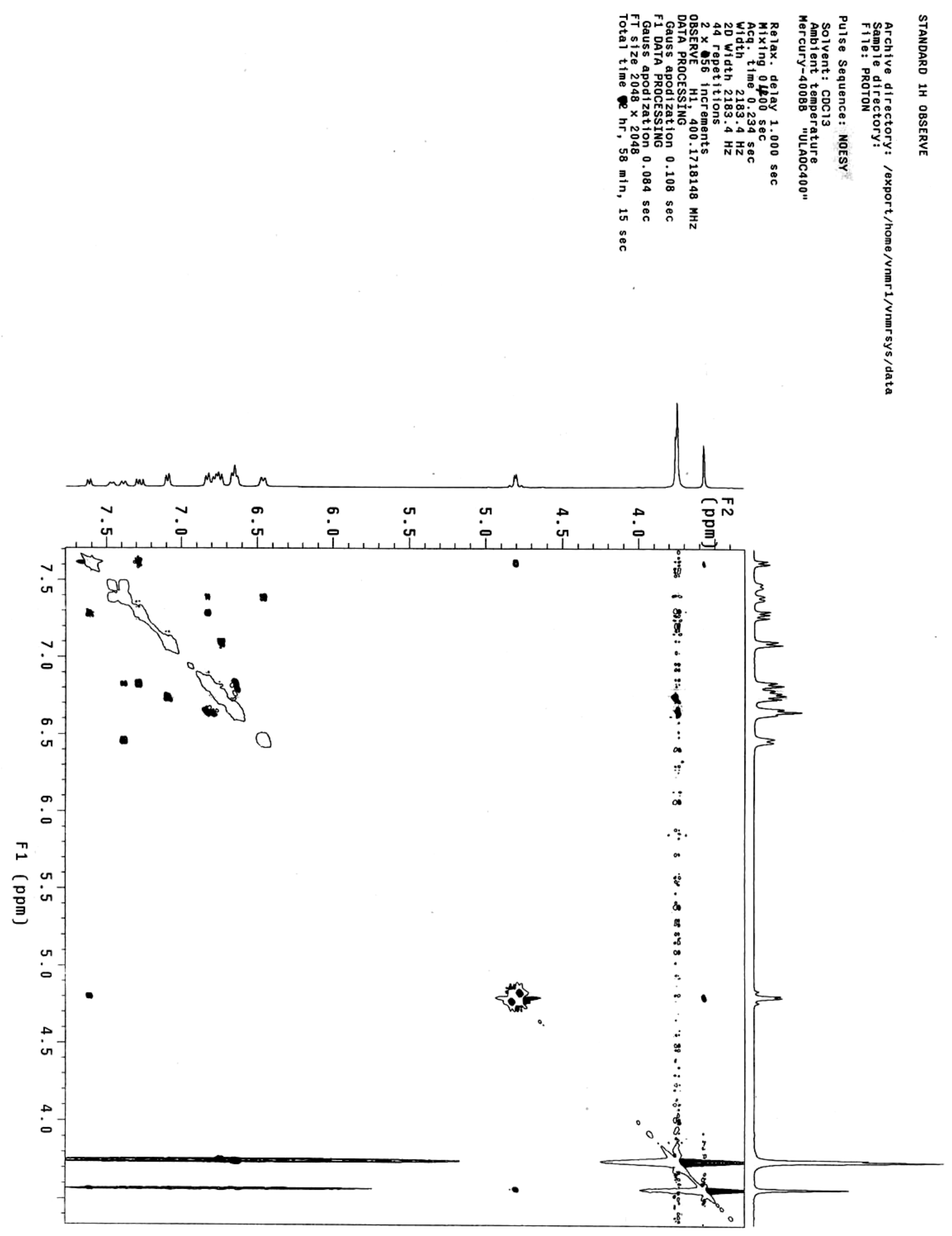




\section{UV-Vis spectrum of Compound 1a}

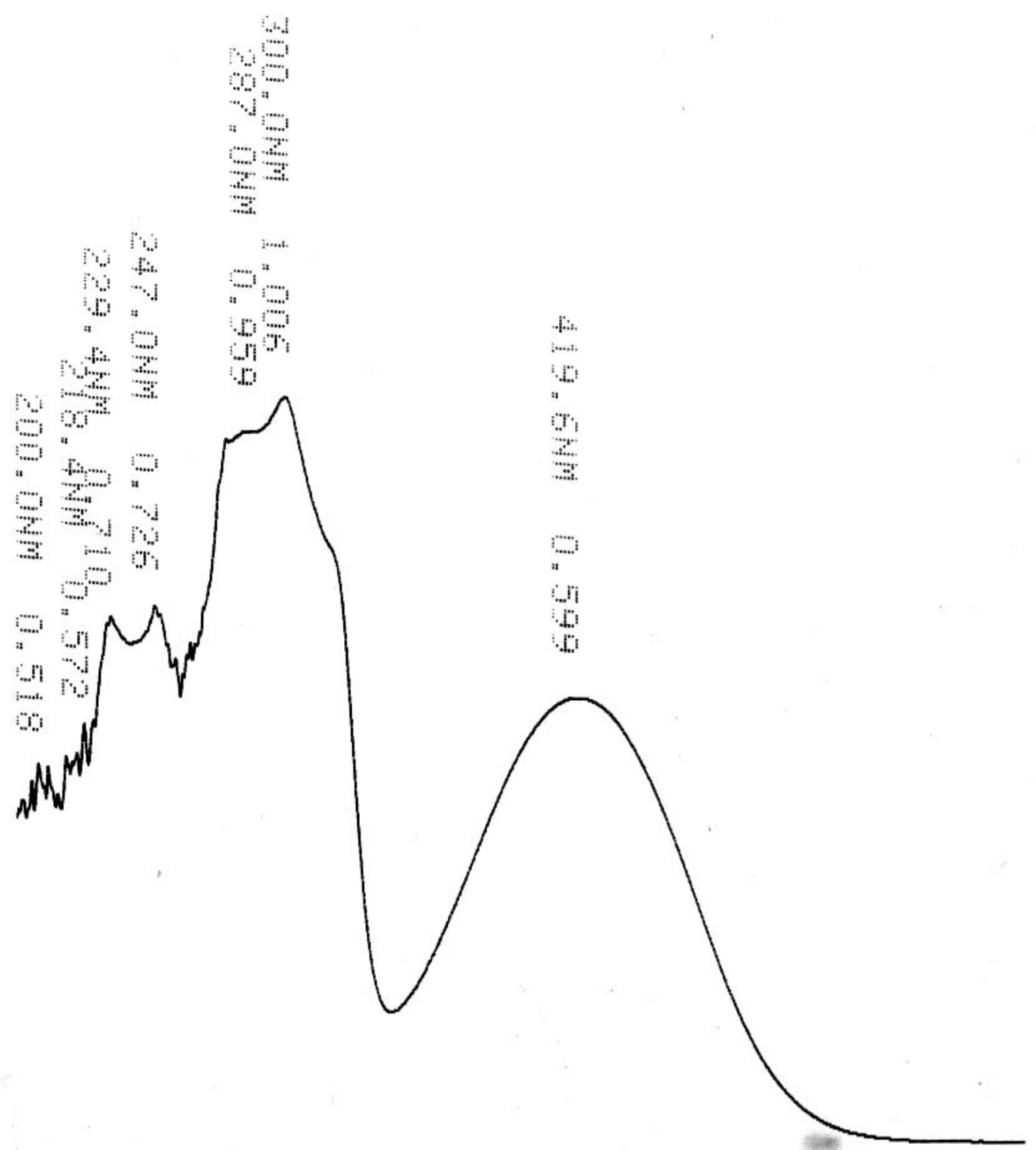

UV-Vis spectrum of Compound 1a in methanol (A versus $\lambda$ ), $\mathrm{c}=9.859 \times 10^{-5} \mathrm{~mol} / \mathrm{L}$ 


\section{ZHANG LP-Subtract-Smooth-Smooth}

Data name: ZHANG LP-Subtract-Smooth-Smooth

File name: $\quad$ C:IProgram FilesIOLIS GlobalWorksIDemo DatalZhangliping-06.5.11LHANG LP-Subtract-Smooth.ols Created: 5/24/2006 9:37:34 AM

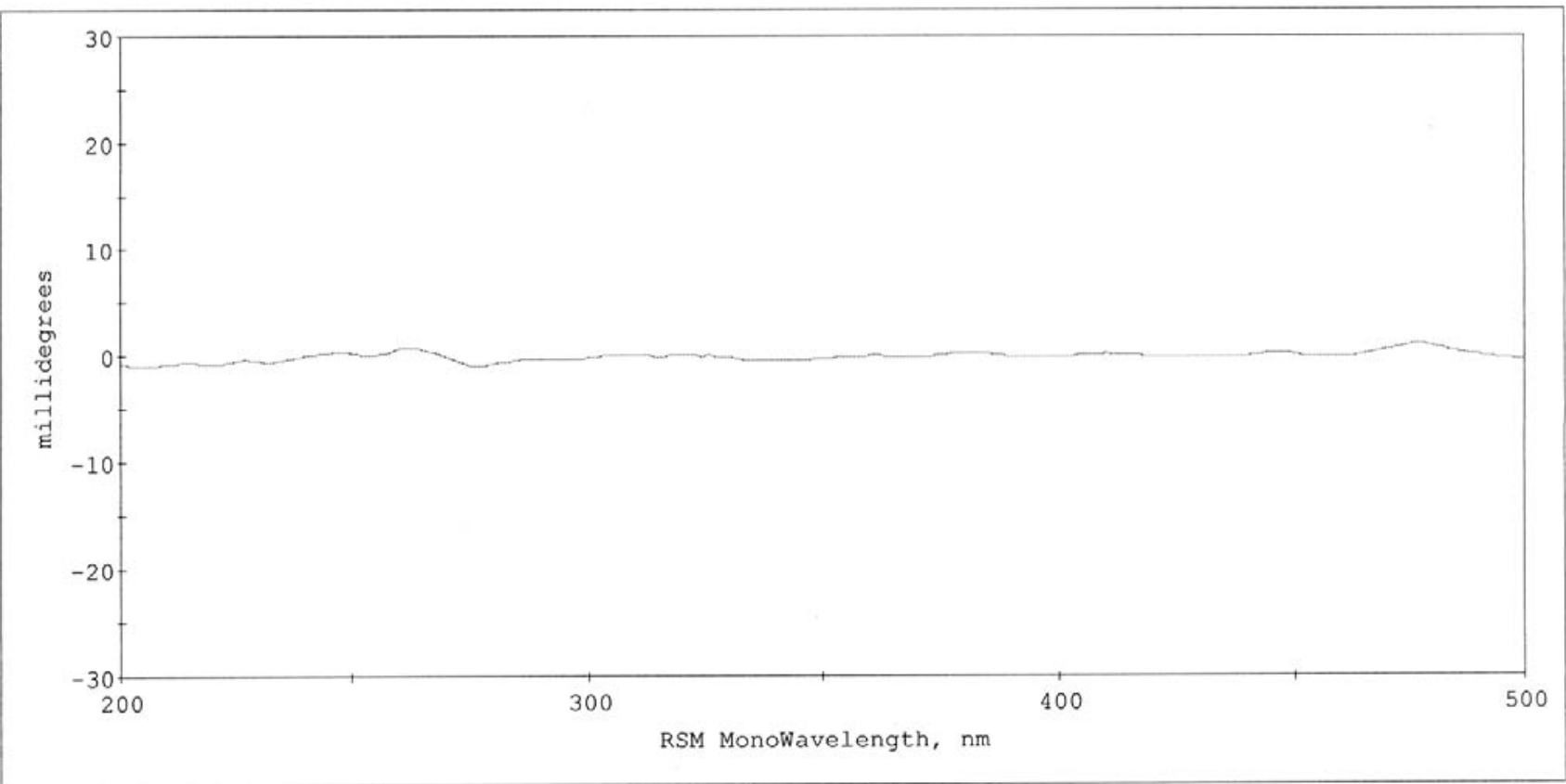

Instrument: RSM

Collection mode: Scan

Number of points: 300

RSM Mono $=500$ to $200 \mathrm{~nm}$ (2400 lines $/ \mathrm{mm}$ )

Timing mode: Constant Time

Integration Time: $0.1 \mathrm{sec}$.

Reduction mode: Circular Dichroism

Scan mode: Fixed slitwidth

(manual Slit width $=$ $\mathrm{mm}$ )

Total Elapsed Time $=3: 20.1$ (min:sec) 
Olis GlobalWorks Data Report

\section{Sample-2-Subtract-Smooth-Smooth}

Data name:

File name:

Sample-2-Subtract-Smooth-Smooth

Created:

C:IProgram FilesIOLIS GlobalWorksIDemo DatalZhangliping-06.5.11106.5.24riCH3OH.ols 5/24/2006 9:37:34 AM

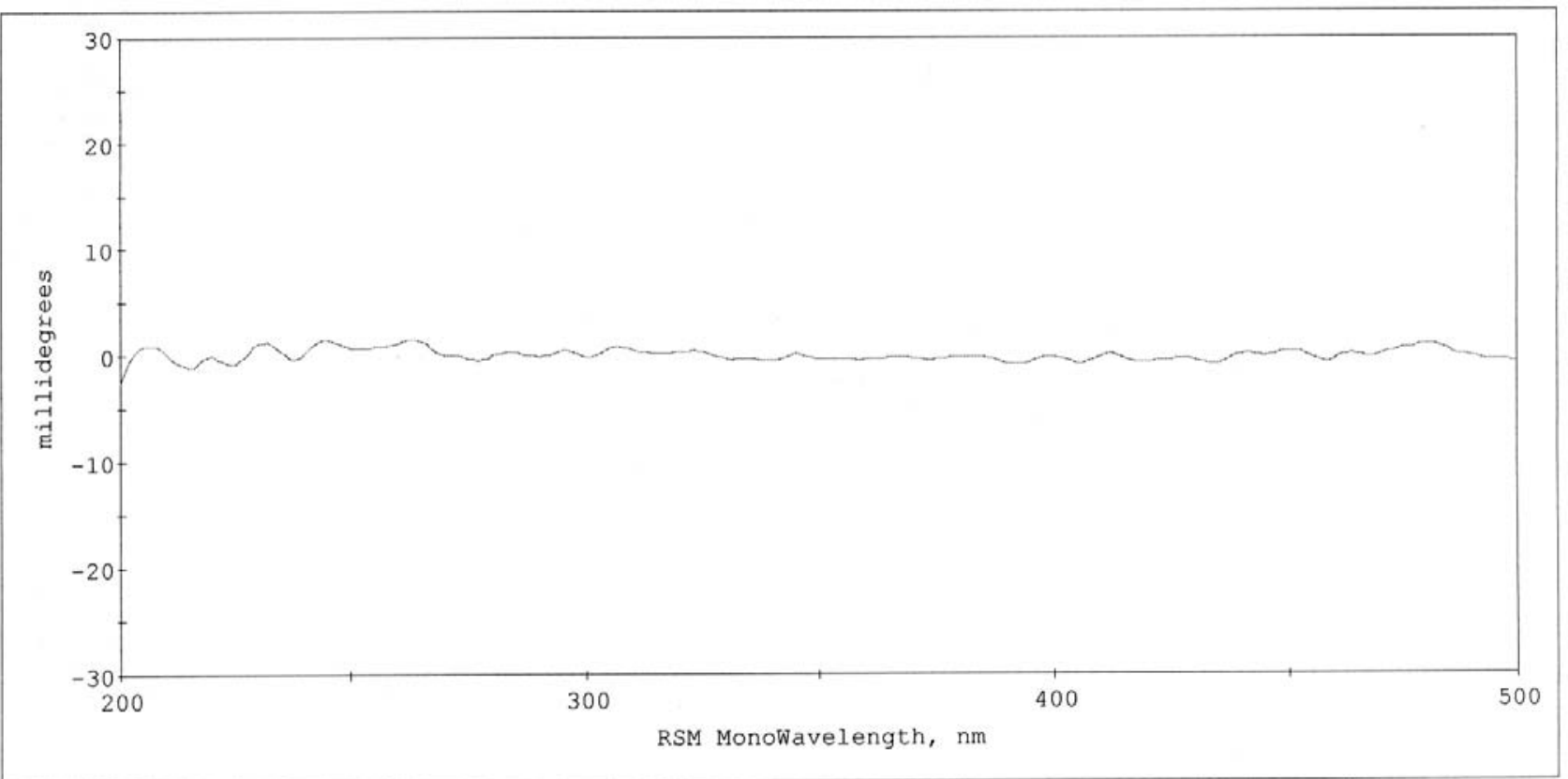

Instrument: RSM

Collection mode: Scan

Number of points: 300

RSM Mono $=500$ to $200 \mathrm{~nm}$ (2400 lines $/ \mathrm{mm}$ )

Timing mode: Constant Time

Integration Time: $0.1 \mathrm{sec}$.

Reduction mode: Circular Dichroism

Scan mode: Fixed slitwidth

(manual Slit width $=$

Total Elapsed Time $=3: 20.1$ (min:sec) $\mathrm{mm})$ 